\title{
P01-1-57
}

Poster session

\section{Selective potentiation of muscarinic acetylcholine receptor subtype 1 demonstrates efficacy and safety in preclinical models of Alzheimer's disease and schizophrenia}

\author{
Jerri M Rook ${ }^{1}$, Jonathan W Dickerson ${ }^{1,2}$, Ryan M Hanson ${ }^{1,2}$, Craig W Lindsley ${ }^{1,2,3}$, P Jeffrey Conn ${ }^{1,2}$ \\ ${ }^{I}$ Pharmacology, Vanderbilt University, USA, ${ }^{2}$ Vanderbilt Center for Neuroscience Drug Discovery, USA, ${ }^{3}$ Chemistry, \\ Vanderbilt University, USA
}

A significant number of preclinical behavioral and human clinical studies provide strong evidence that enhanced cholinergic transmission or activation of muscarinic acetylcholine receptors (mAChRs), notably the M1 subtype, have exciting therapeutic potential for the treatment of cognitive impairments of Alzheimer's disease (AD) and schizophrenia. However, previous M1 activators have failed in clinical development due to a lack of true specificity for M1 and adverse effects associated with activation of other mAChR subtypes (M2-M5). Recently, our group has developed a highly potent, selective series of M1 positive allosteric modulators (PAMs) with enhanced physiochemical and pharmacokinetic properties for in vivo studies, providing an unprecedented opportunity to evaluate the potential of selective potentiation of M1 as a novel target for the treatment of symptoms associated with diminished cognitive function. As opposed to direct activation of M1, PAMs dramatically potentiate the response of the receptor to its endogenous ligand acetylcholine and offer high selectivity while avoiding unwanted side-effects seen with direct activation. Optimized M1 PAMs have been rigorously characterized to establish their potency and efficacy at M1 and excellent selectivity versus other mAChR subtypes. In addition, ancillary pharmacology depicts a clean profile at other clinically relevant receptors. Pharmacokinetic (PK) studies in rats and nonhuman primates demonstrate ideal oral bioavailability and CNS exposure. Moreover, behavioral studies confirm robust cognition enhancing efficacy in multiple preclinical rodent models of both hippocampaland cortical-mediated cognitive function. In safety pharmacology and toxicology studies, no adverse effects were observed in either rodents or NHPs. Finally, a Phase I clinical trial is currently being conducted at Vanderbilt University with our lead clinical candidate to advance a potential new drug for the treatment of cognitive deficits associated with $\mathrm{AD}$ and schizophrenia. 